University of Nebraska - Lincoln

DigitalCommons@University of Nebraska - Lincoln

CSE Conference and Workshop Papers

Computer Science and Engineering, Department of

2013

Exploiting Soil Moisture Information for Adaptive Error Control in Wireless Underground Sensor Networks

Xin Dong

University of Nebraska-Lincoln, xdong@cse.unl.edu

Mehmet C. Vuran

University of Nebraska-Lincoln, mcvuran@cse.unl.edu

Follow this and additional works at: http://digitalcommons.unl.edu/cseconfwork

Dong, Xin and Vuran, Mehmet C., "Exploiting Soil Moisture Information for Adaptive Error Control in Wireless Underground Sensor Networks" (2013). CSE Conference and Workshop Papers. 248.

http://digitalcommons.unl.edu/cseconfwork/248

This Article is brought to you for free and open access by the Computer Science and Engineering, Department of at DigitalCommons@University of Nebraska - Lincoln. It has been accepted for inclusion in CSE Conference and Workshop Papers by an authorized administrator of

DigitalCommons@University of Nebraska - Lincoln. 


\title{
Exploiting Soil Moisture Information for Adaptive Error Control in Wireless Underground Sensor Networks
}

\author{
Xin Dong Mehmet C. Vuran \\ Cyber-Physical Networking Laboratory \\ Department of Computer Science \& Engineering \\ University of Nebraska-Lincoln, Lincoln, NE 68588 \\ Email: \{xdong, mcvuran\}@cse.unl.edu
}

\begin{abstract}
Wireless underground sensor networks (WUSNs) have recently been investigated for a wide range of applications. One challenge in wireless underground communications is its high bit error rate, especially at long distances. In WUSNs, the communication quality is substantially affected by the environment, especially the soil moisture. Thus, the underground channel quality can be effectively estimated based on local soil moisture readings. By utilizing the local soil moisture values to estimate the channel quality, adaptive error control mechanisms can be implemented for underground nodes. In this paper, two error control mechanisms, adaptive-rate forward error control and adaptive transmit power control, are considered for WUSNs. The results indicate that compared to $A R Q$, adaptive FEC code can increase the ranges of soil moisture values within which the network is reliable by reducing the bit error rate. In addition, adaptive transmit power control can improve energy efficiency when a wide range of transmit power levels is available. Our evaluations show that to achieve $60 \mathrm{~m}$ communication distance in practical soil settings, the output power of the transmitter should to be adjusted in a range of $0 \mathrm{dBm}$ to $25 \mathrm{dBm}$ to improve the energy efficiency of the underground nodes.
\end{abstract}

\section{INTRODUCTION}

Wireless underground sensor networks (WUSNs) have recently been investigated for unattended soil monitoring [1], [2], [5], [15], [19]. These networks consist of underground sensor nodes that monitor soil conditions and aboveground base stations that are wirelessly connected to the underground nodes to obtain data and relay them to the end users. Compared with satellites and aerial remote sensing, WUSNs can provide more efficient and precise information about some of the soil conditions, such as soil moisture, at a far less operation cost.

The main challenges of employing WUSNs are two-fold: First, the energy consumption of the underground devices needs to be carefully budgeted since it is not practical to change the batteries [9]. The second challenge is to improve communication quality considering the impediments caused by the soil environment [19]. In [5], we have shown that by designing an antenna according to the propagation characteristics of electromagnetic waves in soil, the communication distance can be significantly improved. However, our testbed experiments show that underground communications suffer from high packet error rates. The impacts of burial depth, packet size, and soil moisture on packet error rate are illustrated in [16]. Therefore, error control schemes need to be adopted to overcome high packet error rates, especially at long distances $(50-80 \mathrm{~m})$ and essentially, improve the feasibility of WUSN applications. More importantly, the error control scheme needs to be energy efficient such that the lifetime of the buried nodes can be extended.

In wireless communications, four mechanisms are exploited to address the problem of packet errors: Automatic RepeatreQuest (ARQ), Forward Error Correction (FEC), Hybrid-ARQ, and adaptive transmit power control [19]. In WUSNs, the energy efficiency of these mechanisms is of interest. Moreover, an efficient error control scheme needs to be designed considering the characteristics of the underground communication channel, especially the change in the soil moisture and the communication distance between the underground and aboveground nodes. Due to the natural precipitation process during the year and irrigation operations for agriculture, soil moisture varies significantly. For an agriculture field, throughout the year, the volumetric water content (VWC) of the field varies between $15 \%$ and $40 \%$. This characteristic of soil causes high variations in the communication quality in wireless underground communications. For example, the packet error rate increases from less than $1 \%$ to more than $50 \%$ when the soil moisture increases from $20 \%$ to $40 \%$ [5]. This calls for error control schemes that are adaptive to the variations in soil moisture.

For FEC codes, adaptability can be achieved through puncturing the generated code words based on the communication channel quality [17]. The rate of the code increases when bits in the code word are punctured. Correspondingly, the error correction capability of the punctured code decreases. Rate-compatible adaptive FEC codes have been implemented using convolutional codes, BCH codes, turbo codes, and LDPC codes [10]. However, it is shown that rate-compatible convolutional codes and $\mathrm{BCH}$ codes do not achieve near capacity performance and rate-compatible turbo codes suffer from high decoding complexity [10]. On the other hand, rate-compatible low density parity check (LDPC) codes have been shown comparable performance to turbo codes but with much less decoding complexity [12]. Due to these favorable characteristics, we are interested in employing LDPC codes to combat the dynamic changes in the underground communication channel caused by variations in soil moisture and communication distance.

Generally, for the application of rate-compatible LDPC 
codes, the transmitter needs to have the knowledge of the communication channel quality in real time. Accordingly, the corresponding error control code rate is chosen to maximize channel capacity [6]. The channel quality information is usually obtained by using feedback packets from the receiver in traditional wireless communications. However, in WUSNs, the communication quality is substantially affected by the environment, especially soil moisture [3], [19]. Thus, the underground channel quality can be estimated based on local soil moisture readings. In other words, the channel estimation can be performed without feedback packets from the receiver.

With the local estimation of the communication channel, transmit power control can also be employed such that the signal to noise ratio (SNR) at the receiver is maintained above a threshold such that the bit error rate is limited. The advantage of adaptive transmit power control is that redundant bits are not needed. However, this may not be energy efficient in terms of energy per bit because even though retransmissions are avoided, the overall energy consumption of each packet increases with higher transmit power.

To the best of our knowledge, the energy efficiency of error control mechanisms under highly varying underground channel conditions due to soil moisture changes is not well known. In the following, the energy efficiency of error control mechanisms for underground communications is investigated. More specifically, adaptive rate-compatible FEC codes and adaptive transmit power control schemes are employed to address the variable packet errors in wireless underground communications. The rate of the FEC code and the transmit power are determined based on local soil moisture measurements and the corresponding communication channel estimation. The energy efficiency of the two schemes are analyzed and compared.

The rest of the paper is organized as follows. The related work is introduced in Section II. The adaptive error control scheme for the underground-to-aboveground communication is described in Section III, including channel model, channel estimation, error control code rate selection and transmit power selection. The energy consumption of the error control mechanisms are discussed in Section IV. Finally, conclusions are drawn in Section V.

\section{RELATED WORK}

For over-the-air communication in sensor networks, the energy efficiency of error control coding is analyzed in [7], [18], where the gain in energy saving at the transmitter at the cost of decoder power consumption at the receiver is studied. It is shown in [7] that in lossy environments and in high frequency situations, the benefits of error control outweigh the cost. Since underground communication has a higher error rate than its over-the-air counterpart, these results motivate the investigation of error control schemes for underground communications. In [18], three kinds of error control schemes: FEC, ARQ and Hybrid-ARQ, are evaluated in a cross-layer analysis. FEC schemes improve the error resiliency compared to ARQ and this improvement can be exploited by reducing the transmit power or by constructing longer distance communication. However, variations in the communication channel are not considered in [18], which is a major challenge for wireless underground

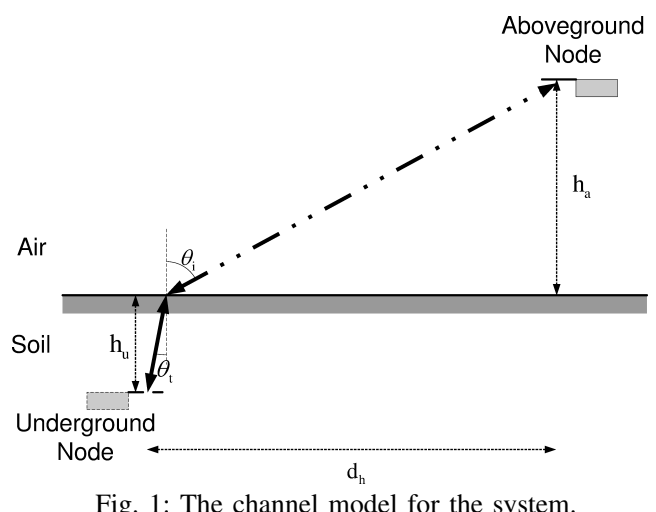

communication. For underground communication, we also empirically investigated these two error control schemes [16], where the impacts of burial depth, packet size and soil moisture on packet error rate are analyzed. However, adaptive FEC codes as well as transmit power control have not been considered for WUSNs yet.

Adaptive FEC codes are usually limited to Hybrid-ARQ schemes, where based on the ARQ packets from the receiver, the transmitter sends more redundant data to help the receiver correctly decode the packet [6], [10]. In WUSNs, the environment properties, which affect communication quality, can be measured at the transmitter. This provides a unique way to adjust the error control rate at the transmitter without a feedback from the receiver. To the best of our knowledge, the employment of the adaptive FEC codes in WUSNs has never been considered before. In the following, the adaptive LDPC codes and the adaptive transmit power control in underground communications are described in detail.

\section{AdAptive ERror CONTROL FOR Underground COMMUNICATIONS}

In this section, we provide a background on underground communications in Section III-A. Then, underground channel estimation is discussed in Section III-B and the adaptive error control schemes are discussed in Section III-C.

\section{A. Channel Model for Soil-Air Communication}

In WUSNs, sensor nodes are buried in the ground and communicate with aboveground nodes [5]. Thus, the communications can be categorized into two links, the undergroundto-aboveground (UG2AG) link and the aboveground-tounderground (AG2UG) link [13], [14]. Due to the two different situations of refraction at the soil-air interface, the two links are not symmetric. In our previous work, the channel models for these two links have been developed and validated with field experiments [5]. In the following, we summarize our findings in [5], [13] for completeness.

For both AG2UG and UG2AG links, the channel consists of two parts: the underground portion (soil medium) and the aboveground portion (air medium), as shown in Fig. 1. Given the horizontal distance, $d_{h}$, the height of the AG antenna, $h_{a}$, and the burial depth of the UG nodes, $h_{u}$; the length of both portions can be calculated. Accordingly, the received signal strength at the receiver, $P_{r}$, is given as

$$
P_{r}=P_{t}+G_{t}+G_{r}-\left(L_{u g}\left(d_{u g}\right)+L_{a g}\left(d_{a g}\right)+L_{(R, \rightarrow)}\right),
$$


where $P_{t}$ is the transmit power, $G_{t}$ and $G_{r}$ are the antenna gains at the sender and the receiver, respectively. $L_{u g}\left(d_{u g}\right)$ and $L_{a g}\left(d_{a g}\right)$ are the loss at the underground and the aboveground portions, respectively. Finally, $L_{(R, \rightarrow)}$ is the refraction loss based on the propagation direction, $\rightarrow$, i.e., ag $2 u g$ or $u g 2 a g$, which is the main source of asymmetry between the AG2UG and UG2AG links.

The underground and aboveground losses in (1) are given as [19]:

$$
\begin{aligned}
& L_{u g}\left(d_{u g}\right)=6.4+20 \log d_{u g}+20 \log \beta+8.69 \alpha d_{u g}, \\
& L_{a g}\left(d_{a g}\right)=-147.6+10 \eta \log d_{a g}+20 \log f,
\end{aligned}
$$

respectively, where $\eta$ is the attenuation coefficient in air, $f$ is the operation frequency, $\beta$ is the phase shifting constant, and $\alpha$ is the attenuation constant. The attenuation coefficient in air, $\eta$, is higher than 2 due to the impacts of ground reflection. Our empirical experiments show that $\eta$ is in the range of 2.8-3.3 [5]. The impact of soil properties on attenuation are captured by the last two terms in (2), where $\alpha$ and $\beta$ are given as $k_{s}=$ $\alpha+i \beta=i \omega \sqrt{\mu_{0} \epsilon_{s}}$, where $k_{s}$ is the propagation constant in soil, $\mu_{0}$ is the permeability in free space and $\epsilon$ is the effective soil permittivity.

Due to the higher permittivity of soil, electromagnetic waves reflect and refract at the soil-air interface. Signals can penetrate through the interface only if the incident angle is small. For the UG2AG propagation, only the waves with small incident angle $\left(\theta_{t}\right.$ in Fig. 1) will penetrate to air. On the other hand, for the AG2UG propagation, the refracted angle is near to zero and the propagation in soil is also vertical. Thus, for both links, the underground portion of communication distance can be approximated as $d_{u g} \simeq h_{u}$, where $h_{u}$ is the burial depth and the aboveground portion is approximated as $d_{a g}=\sqrt{h_{a}^{2}+d_{h}^{2}}$, where $h_{a}$ is the height of the AG node and $d_{h}$ is the horizontal distance between nodes.

For the AG2UG link, we consider the maximum power path where the incident angle, $\theta_{i} \rightarrow 0$. Thus, the refraction loss, $L_{(R, \rightarrow)}$, in (1) can be approximated as $L_{(R, a g 2 u g)} \simeq 20 \log \frac{n+1}{4}$ [8], where $n$ is the refractive index of soil, which is given by $n=\sqrt{\frac{\sqrt{\epsilon^{\prime 2}+\epsilon^{\prime \prime 2}+\epsilon^{\prime}}}{2}}[5]$.

For the UG2AG link, the signal propagates perpendicularly from a higher density medium to a lower density one. Hence we consider all energy is refracted (i.e., $\left.L_{(R, u g 2 a g)}=0\right)$.

In Fig. 2, the received signal strength at the receiver, calculated from both channel models, is depicted as a function of the distance between the underground node and aboveground node for two soil moisture values. The results are also compared with our test bed results conducted in the South Central Agriculture Laboratory of the University of Nebraska-Lincoln [5]. In the evaluations, the underground node is buried at $0.4 \mathrm{~m}$, the aboveground node is at $2 \mathrm{~m}$ and the transmit power is set at $10 \mathrm{dBm}$. It is shown that the model matches the testbed results in most cases. The maximum difference is less than $2 \%$.

\section{B. Channel Estimation at the Underground Node}

In WUSNs, the aboveground nodes request the underground nodes to collect soil moisture information. It is relatively easy to provide energy to the aboveground nodes (e.g., with solar

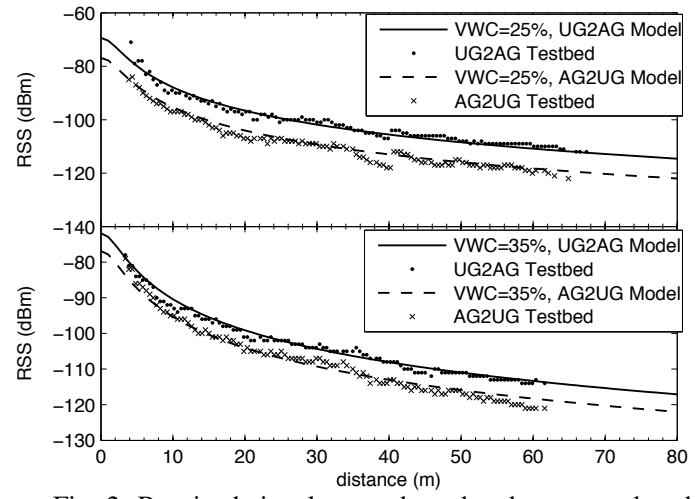

Fig. 2: Received signal strength at the aboveground node.

panels). Therefore, energy consumption at the aboveground nodes is not considered in the following. On the other hand, for the underground nodes, the energy efficiency, in terms of energy per bit, is of major importance. To improve energy efficiency, both adaptive FEC and adaptive transmit power control techniques can be employed. For both schemes, the underground node needs to estimate the quality of the channel. To this end, the underground node can exploit the local soil moisture information to effectively estimate the communication channel.

In typical underground monitoring applications, aboveground node initiates the communication by transmitting a REQUEST packet. After receiving REQUEST, the underground node can estimate the channel quality. More specifically, the received signal strength at the aboveground node is a function of its own transmit power. Comparing AG2UG and UG2AG links based on (1), it can be found that

$$
P_{r a}=P_{t a}+P_{t u}-P_{r u}-L_{(R, a g 2 u g)},
$$

where $P_{t a}$ and $P_{t u}$ are the transmit powers of the aboveground and underground nodes, respectively, $P_{r u}$ is the received power at the underground node, and $P_{r a}$ is the received power at the aboveground node. In (4), the received power at the underground node, $P_{r u}$, can be obtained as from the received signal strength (RSS) readings from the transceiver chip. For the transmit power of the aboveground nodes, $P_{t a}$, two methods can be adopted. First, since energy consumption is not a concern at the aboveground node, it can always utilize the maximum transmit power, thus $P_{t a}$ becomes a constant. On the other hand, even if the aboveground node changes $P_{t a}$, the value can be piggybacked to the REQUEST packets and hence the underground node obtains $P_{t a}$ from the packets. Thus, to estimate the channel, the underground node need to estimate the aboveground to underground refraction loss, $L_{(R, a g 2 u g)}$.

$L_{(R, a g 2 u g)}$ is a function of the soil permittivity, $\epsilon$, which is itself a function of the soil type and soil moisture (measured in volumetric water content). Given a soil type, $\epsilon$ can be modeled using the Peplinski model [11] for different soil moisture levels. Thus, by sensing the local soil moisture, the underground nodes can calculate $\epsilon$ and hence the refraction loss, $L_{(R, a g 2 u g)}$. As a result, the underground nodes can estimate the received power at the aboveground node as a function of its own transmit power.

In most WUSN applications, the devices are deployed in 


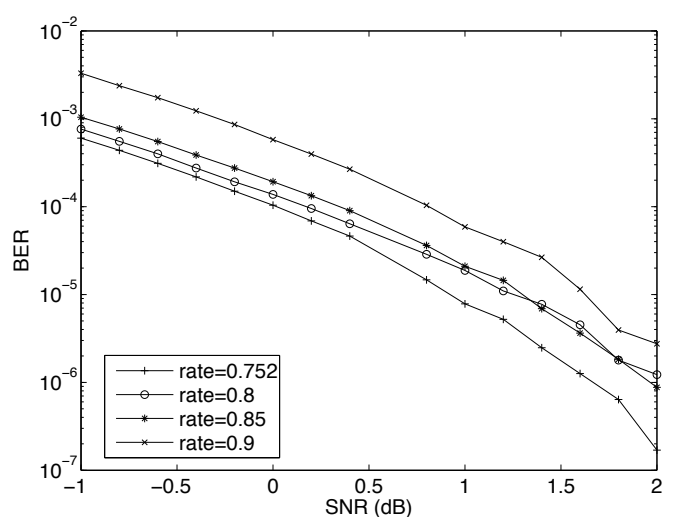

Fig. 3: Bit error rate of the LDPC code at different rate.

open fields where interference is negligible and the thermal noise is the main source of noise [13]. In our analysis, the noise floor, $N$, is determined and thus the signal noise ratio is calculated as $\mathrm{SNR}=P_{r a}-N$.

\section{Adaptive FEC Rate and Adaptive Transmit Power}

Based on the estimated signal strength at the aboveground node, the underground node can determine the best strategy to successfully transmit the packet in terms of energy efficiency. For adaptive FEC code, the FEC code rate can be determined and the generated code-word can be punctured according to the pre-calculated puncturing index.

In most WUSN applications (e.g., the autonomous center pivot irrigation [5]), the aboveground node collects soil moisture data from an underground node periodically (usually with a period of more than a week) and the underground node sends the soil moisture data for the whole week. Thus, the length of the information data is in hundreds to thousands of bits. The rate compatible LDPC code evaluated in this paper is generated using the algorithm described in [17]. The base code is 1212 bit long, and the information section is 911 bit long. Thus, the size of the parity check matrix, $H$, is $1212 \times 301$.

By applying the puncturing algorithm described in [17], the useful rate of the rate-compatible LDPC, denoted as $r_{E C}$, is found to be in the range of 0.75 to 0.9 . In other words, up to 200 bits can be punctured to generate a high rate code word.

The performance of the rate-compatible LDPC code, as measured by the bit error over signal noise ratio, is shown in Fig. 3. It is observed that for a BER target of $10^{-4}$, there is a $0.8 \mathrm{~dB}$ difference in the SNR requirement between the highest and lowest rates.

In the evaluations, a threshold, $\Delta$, is chosen such that if the BER is less than $\Delta$, the packet is considered successfully transmitted. Otherwise, the transmission of the packet fails. To compare the energy efficiency of different rates, the energy efficiency as energy per bit is calculated as $E_{i}=\frac{P_{u t}}{R \cdot r_{E C}}$ [18], where $P_{u t}$ is the transmit power, $R$ is the data rate and $r_{E C}$ is the rate of the LDPC code.

In cases where the transmit power of the underground node cannot be changed, the optimal rate of the FEC code is found as an optimization problem:

$$
r_{E C}^{*}=\underset{r_{E C}}{\arg \min } \frac{P_{u t}}{R \cdot r_{E C}},
$$

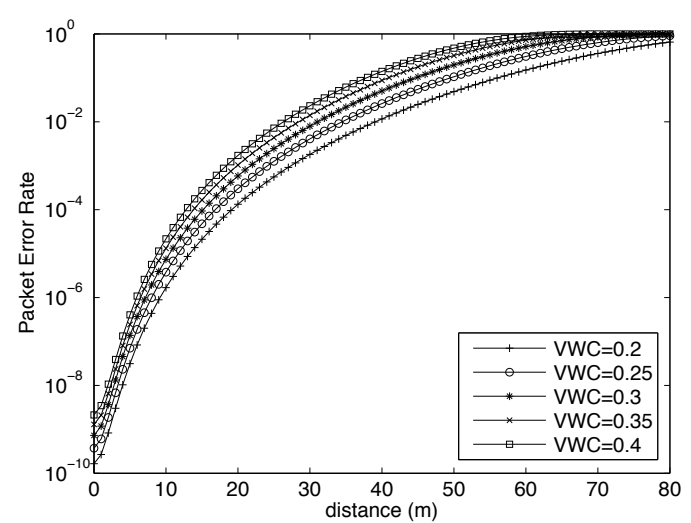

Fig. 4: Packet error rate at the aboveground node.

subject to

$$
\text { BER }<\Delta,
$$

where BER is the bit error rate when the FEC code rate is $r_{E C}$ and is obtained by simulation.

In cases where adjusting transmit power, $P_{u t}$, is also possible, the adaptive error control scheme needs to consider both the transmit power and the rate of the LDPC code. Thus, the best scheme is found by solving the two-variable optimization problem:

$$
\left[P_{u t}^{*}, r_{E C}^{*}\right]=\underset{P_{u t}, r_{E C}}{\arg \min } \frac{P_{u t}}{R \cdot r_{E C}} .
$$

Due to the fact that the BER of the FEC code is obtained by simulation, the optimization problem is solved in discrete domain. In other words, the transmit power, $P_{u t}$ is discretized. This is not an issue in practice since the output power of the transmitter is not continuous. To solve this problem, $E_{i}$ is calculated for all the combinations of $P_{u t}$ and $r_{E C}$ and the optimal parameter set is found. Note, the underground nodes do not need to solve the optimization problem online. Instead, the schemes are pre-calculated offline and stored at the underground node as a look-up table. Based on soil moisture measurement and SNR estimation, the underground node chooses $P_{u t}$ and $r_{E C}$ from the table.

The energy consumption analyses of both the cases are discussed in Section IV.

\section{EnERgy CONSUmption ANALysis}

In this section, we analyze the energy consumption of the adaptive error control schemes. In our testbeds, Mica2 motes are employed due to their low operating frequency $(433 \mathrm{MHz})$ which suffers less attenuation in soil compared to the $2.4 \mathrm{GHz}$ frequency used by MicaZ motes. Thus, in this section, the analyses are based on Mica2 motes. Note, the method used developed in Section III is not limited to Mica2 and can be easily applied to other platforms according to the operation frequency and modulation scheme. The modulation scheme in Mica2 is non-coherent FSK modulation, for which the bit error rate is given by $p_{b}^{F K S}=\frac{1}{2} e^{-\frac{E_{b} / N_{0}}{2}}$, and $E_{b} / N_{0}=\psi \frac{B_{N}}{R}$, where $\psi$ is the SNR, $B_{N}$ is the noise bandwidth and $R$ is the data rate. In addition, the packet error rate (PER) of a packet without FEC of length $l$ is given by $\delta_{F K S}(l)=1-\left(1-p_{b}^{F K S}\right)^{l}$. 


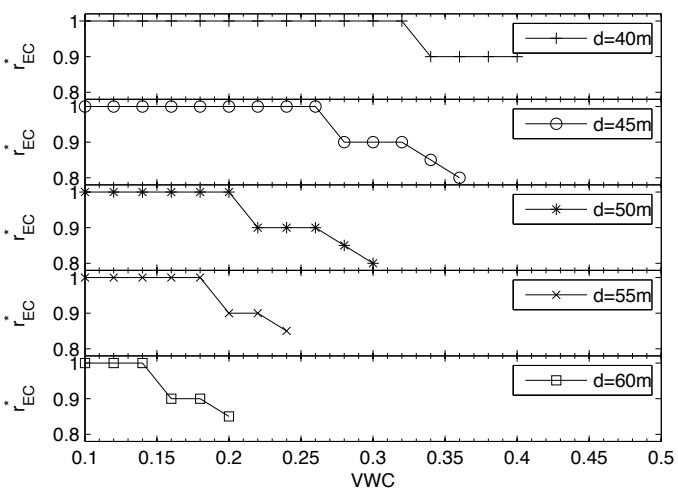

(a)

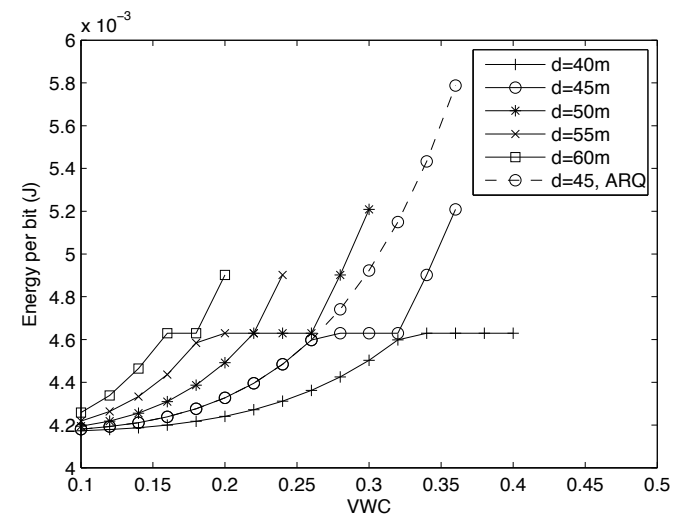

(b)

Fig. 5: The optimal rate of the LDPC code and the corresponding energy efficiency for the transmit power of $10 \mathrm{dBm}$.

In Fig. 4, the PER as a function of distance between underground node and aboveground node for different soil moisture levels is shown, where the packet size is 911 bits, the transmit power is $10 \mathrm{dBm}$ and the noise level is $-110 \mathrm{dBm}$. It is shown that PER increases very rapidly with distance. Moreover, due to the different PERs at different soil moisture values, the communication distance between the underground and aboveground nodes varies when soil moisture changes. For a VWC value of $20 \%$, the reliable communication distance for a PER threshold of $10^{-2}$ is $40 \mathrm{~m}$. If the VWC increases to $25 \%$, the communication distance decreases to $35 \mathrm{~m}$ and a further increase in VWC to $40 \%$ reduces the communication distance to $26 \mathrm{~m}$. Consequently, a $100 \%$ increase in VWC leads to a $35 \%$ decrease in the communication distance.

IF FEC is not adopted, retransmission is needed for overcoming bit errors, which may decrease the energy efficiency of the transmitter. In the following, energy efficiency improvement by the adaptive error control schemes is analyzed, especially at the far distances $(40 \mathrm{~m}$ to $60 \mathrm{~m}$ ).

\section{A. Adaptive FEC with Fixed Transmit Power}

In some applications, it may not be feasible or desirable to adjust the transmit power of the underground nodes. Thus, the underground nodes can only adjust the FEC code rate to adopt to the dynamics of the channel. In Fig. 5, the results where the transmit power is set at $10 \mathrm{dBm}$ are shown, where the bit error threshold, $\Delta$, is set at $10^{-4}$. In Fig. 5(a), the optimal rates of the LDPC code as a function of the VWC are shown for five different communication distances. In the figures, a rate of 1 indicates that the packet is sent without FEC.

It is observed that when soil moisture increases, a lower LDPC code rate is needed to compensate for the increasing bit errors. For example, for a distance of $45 \mathrm{~m}$, the FEC code is used when the VWC is in the range of $28 \%$ to $36 \%$. The rate decreases from 0.9 to 0.85 when VWC increases from $32 \%$ to $34 \%$, and it further decreases to 0.80 when VWC increases to $36 \%$. When VWC increases further, no FEC rates are chosen. This is because at these situations, the bit error rate becomes greater than the threshold $\Delta$ and packet errors occur even when FEC code is applied. Therefore, for far distances (e.g., $60 \mathrm{~m}$ ), the communication occurs only when the soil is dry (VWC is less than 20\%).

In Fig. 5(b), the energy per bit is shown. For comparison, the case when ARQ is used at the distance of $45 \mathrm{~m}$ is also shown. It is observed that for a given VWC, FEC code reduces energy per bit since retransmissions are not required. For a distance of $45 \mathrm{~m}$ and VWC of $30 \%$, the energy per bit for the FEC code is $4.63 \mathrm{~mJ}$, compared to the $4.92 \mathrm{~mJ}$ of ARQ, a $5.9 \%$ decrease. Moreover, if the energy per bit is limited at $5 \mathrm{~mJ}$, by adopting FEC code, communications at $45 \mathrm{~m}$ can be successful at a VWC of $34 \%$, compared to $30 \%$ if ARQ is used. Also observed in Fig. 5(b) is that when FEC code is adopted, energy per bit increases when a lower rate is employed. Since a lower rate means more redundant bits, the energy efficiency reduces. However, the price of increasing redundant bits is still less than the penalty of retransmission, as shown in the $d=45 \mathrm{~m}$ case.

\section{B. Adaptive FEC and Transmit Power}

In cases where the transmit power of the underground nodes can be adjusted, in addition to finding the optimal rate for the FEC code, the transmit power can also be adjusted such that the SNR at the aboveground is above a certain value and a low packet error rate is achieved. In Fig. 6, the numerical evaluation results of adjusting both FEC code rate and transmit power are depicted. In all the cases, no FEC is used in the best strategy and hence only the results of transmit power are shown.

As shown in Fig. 6(a), to achieve the optimal energy efficiency, the transmitter needs to support a wide range of output power. The lowest transmit power, $0 \mathrm{dBm}$, occurs when the communication distance is $1 \mathrm{~m}$ and the VWC is $20 \%$. On the other hand, the highest transmit power, $21.51 \mathrm{dBm}$, occurs when the communication distance is $50 \mathrm{~m}$ and the VWC is $40 \%$. The energy per bit also varies significantly as shown in Fig. 6(b), from $2.121 \times 10^{-7} \mathrm{~J}$ to $0.01344 \mathrm{~J}$. Thus, the maximum energy per bit is more than 60000 times than the lowest energy per bit. In all the cases, the packet error rate is less than $10^{-3}$. Thus the chance of retransmissions is very low. Note that the theoretical analysis may not be applicable to practical transceiver chips as the output range of a transceiver may not be as large as required. For example, the transceiver used in Mica2, $\mathrm{CC} 1000$, has an output power of $-20 \mathrm{dBm}$ to $10 \mathrm{dBm}$, which can only be changed in 10 steps. Similarly, the dependence of power amplifier efficiency on the transmit power level is not considered in the analysis, which may hamper the advantages of transmit power control solutions in practice. 


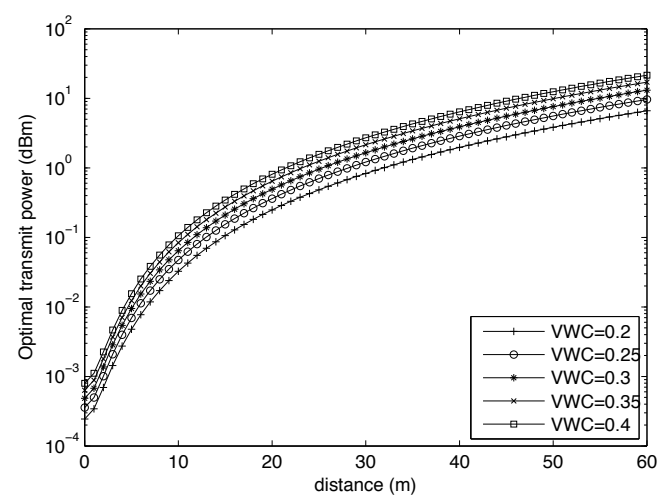

(a)

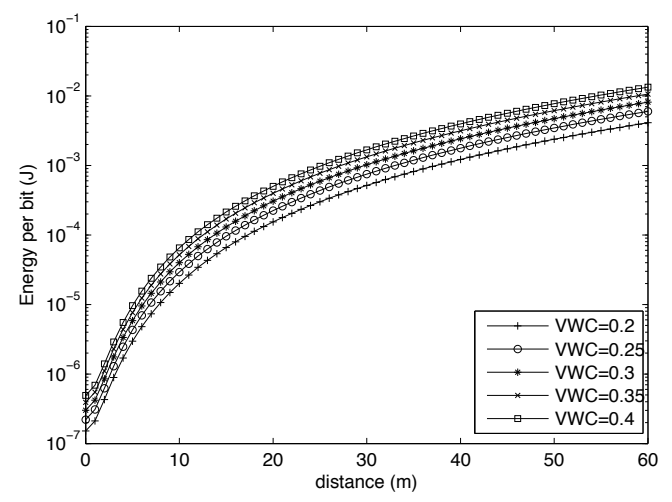

(b)

Fig. 6: The optimal transmit power and the corresponding energy efficiency.

In our evaluations, we did not consider the possible collisions of the packets at the aboveground nodes. As shown in our previous research [4], the UG2AG channel suffers from hidden terminal problems because the transmission ranges of the UG2UA channel is substantially longer than the UG2UG channel $(50 \mathrm{~m}$ vs. $5 \mathrm{~m})$. With the increase in underground node transmit power, collision rates increase as well. As shown empirically in [4], the underground nodes need to contend for the channel up to three times before sending a packet. In this case, transmit power control may not be desirable to combat the increasing soil moisture as the interference range may increase, causing collisions in network settings. In cases where the transmit power should be limited, adaptive FEC codes are desirable, as shown in Section IV-A. This trade off is out of the scope of this paper but deserves further investigation.

\section{CONCLUSIONS}

In this paper, adaptive error control mechanisms are analyzed for wireless underground sensor networks. The underground channel quality is highly correlated with the soil environment, especially soil moisture. Thus, the underground node can effectively estimate the channel quality by measuring the local soil moisture. Two error control mechanisms, adaptive FEC code and adaptive transmit power control, are considered. The results indicate that when the transmit power cannot be changed, adaptive FEC code increases energy efficiency by reducing packet error rate. Compared to $\mathrm{ARQ}$, the energy per bit can be reduced up to $10 \%$. Moreover, when transmit power can be changed, adaptive transmit power control is more efficient when energy consumption is considered. Our numerical evaluation shows that to achieve $60 \mathrm{~m}$ communication distance in a practical soil setting, the output power of the transmitter needs to be adjustable in the range of $0 \mathrm{dBm}$ to $25 \mathrm{dBm}$ to achieve the optimal energy efficiency.

\section{ACKNOWLEDGMENTS}

This work is supported by an NSF CAREER award (CNS0953900) and an NSF I-Corps grant (IIP-1248926).

\section{REFERENCES}

[1] I. F. Akyildiz and E. P. Stuntebeck, "Wireless underground sensor networks: Research challenges," Ad Hoc Networks Journal (Elsevier), vol. 4, pp. 669-686, July 2006.

[2] H. R. Bogena, J. A. Huismana, H. Meierb, U. Rosenbauma, and A. Weuthena, "Hybrid wireless underground sensor networks: Quantification of signal attenuation in soil," Vadose Zone Journal, vol. 8, no. 3, pp. 755-761, August 2009.

[3] X. Dong and M. C. Vuran, "Environment aware connectivity for wireless underground sensor networks," in Proc. INFOCOM '13, Turin, Italy, Apr. 2013.

[4] — , "Empirical analysis of the hidden terminal problem in wireless underground sensor networks," in International Conference on Wireless Communications in Unusual and Confined Areas (ICWCUCA), Aug. 2012.

[5] X. Dong, M. C. Vuran, and S. Irmak, "Autonomous precision agricultrue through integration of wireless underground sensor networks with center pivot irrigation systems," Ad Hoc Networks (Elsevier), 2013, in Press.

[6] J. Ha, J. Kim, D. Klinc, and S. W. McLaughlin, "Rate-compatible punctured low-density parity-check codes with short block lengths," IEEE Transactions on Information Theory, vol. 52, no. 2, pp. 728-738, Feb. 2006.

[7] S. L. Howard, C. Schlegel, and K. Iniewski, "Error control coding in lowpower wireless sensor networks: when is ecc energy-efficient?" EURASIP Journal on Wireless Communications and Networking, vol. 2006, no. 2, pp. 29-29, Feb. 2006.

[8] C. T. Johnk, Engineering Electromagnetic Fields and Waves, 2nd ed. John Wiley \& Sons, Jan. 1988.

[9] S. Kahrobaee and M. C. Vuran, "Vibration energy harvesting for wireless underground sensor networks," in Proc. IEEE ICC '13, Budapest, Hungary, Jun. 2013.

[10] J. Li and K. Narayanan, "Rate-compatible low density parity check codes for capacity-approaching arq scheme in packet data communications," in International Conference on Communications, Internet, and Information Technology (CIIT), St. Thomas, US Virgin Islands, Nov. 2002, pp. 201206.

[11] N. Peplinski, F. Ulaby, and M. Dobson, "Dielectric properties of soil in the 0.3-1.3 ghz range," IEEE Transactions on Geoscience and Remote Sensing, vol. 33, no. 3, pp. 803-807, May 1995.

[12] T. J. Richardson and R. L. Urbanke, "The capacity of low-density paritycheck codes under message-passing decoding," IEEE Transactions on Information Theory, vol. 47, no. 2, pp. 599-618, Feb. 2001.

[13] A. R. Silva and M. C. Vuran, "Communication with aboveground devices in wireless underground sensor networks: An empirical study," in Proc. of IEEE ICC'10, Cape Town, South Africa, May 2010, pp. 1-6.

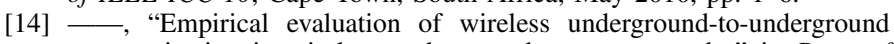
communication in wireless underground sensor networks," in Proc. of IEEE International Conference on Distributed Computing in Sensor Systems (DCOSS '09), Marina del Rey, CA, June 2009, pp. 231-244.

[15] M. J. Tiusanen, "Attenuation of a Soil Scout radio signal," Biosystems Engineering, vol. 90, no. 2, pp. 127-133, January 2005.

[16] J. Tooker and M. C. Vuran, "Mobile data harvesting in wireless underground sensor networks," in Proc. IEEE SECON '12, Seoul, Korea, June 2012.

[17] B. N. Vellambi and F. Fekri, "Finite-length rate-compatible ldpc codes: A novel puncturing scheme," IEEE Transactions on Communications, vol. 57, no. 2, pp. 297-301, Feb. 2009.

[18] M. C. Vuran and I. F. Akyildiz, "Error control in wireless sensor networks: a cross layer analysis," IEEE/ACM Transactions on Networking, vol. 17, no. 4, pp. 1186-1199, 2009.

[19] _ - "Channel model and analysis for wireless underground sensor networks in soil medium," Physical Communication, vol. 3, no. 4, pp. 245-254, December 2010. 